\title{
Stenotrophomonas strain CPCC 101271, an intestinal lifespan-prolonging bacterium for Caenorhabditis elegans that assists in host resistance to "Bacillus nematocida" colonization
}

\author{
Rui Han ${ }^{1} \cdot$ Yu Wang ${ }^{1} \cdot$ Yang Deng $^{2} \cdot$ Yuqin Zhang $^{2} \cdot$ Lin Zhang $^{1} \cdot$ Qiuhong Niu ${ }^{1}$ (I)
}

Received: 7 May 2021 / Revised: 24 June 2021 / Accepted: 25 June 2021 / Published online: 14 July 2021

(C) The Author(s) 2021

\begin{abstract}
The soil-dwelling, opportunistic pathogenic bacterium "Bacillus nematocida" B16 exhibits strong killing activities against a variety of pathogenic nematodes via a "Trojan horse" mechanism that can kill worm species like Caenorhabditis elegans. The bacterial strain CPCC 101271 was previously isolated from the intestines of C. elegans that were recovered from natural habitats and can serve as a probiotic for $C$. elegans, while also assisting in resistance to infection by the pathogenic strain B16. In this study, the lifespan of C. elegans fed with strain CPCC 101271 cells was extended by approximately $40 \%$ compared with that of worms fed with Escherichia coli OP50 cells. In addition, the colonization of $C$. elegans by the pathogenic bacterium "B. nematocida" B16 was inhibited when pre-fed with strain CPCC 101271. Metagenomic sequence analysis of intestinal microbiota of $C$. elegans fed with strain CPCC 101271 and infected with B16 revealed that pre-feeding worms with CPCC 101271 improved the diversity of the intestinal bacteria. Moreover, community structure significantly varied in coordination with Stenotrophomonas spp. and Bacillus spp. abundances when competition between strains CPCC 101271 and B16 was evaluated. In conclusion, the nematode microbiota strain CPCC 101271 assisted in its host resistance to colonization by the pathogen "Bacillus nematocida" and can also promote life span-prolongation in C. elegans. These results underscore that understanding the interactions between $C$. elegans microbiota and pathogens can provide new insights into achieving effective biological control of agricultural pests.
\end{abstract}

Keywords Stenotrophomonas · Caenorhabditis elegans · "Bacillus nematocida" · Lifespan-prolonging · Colonization

Communicated by Erko Stackebrandt.

Rui Han and Yu Wang contributed equally to the work.

Lin Zhang

zhanglin201207@163.com

$\triangle$ Qiuhong Niu

qiuhongniu723@163.com

1 College of Life Science and Agricultural Engineering, Nanyang Normal University, 1638 Wolong Road, Nanyang 473061, Henan, China

2 Institute of Medicinal Biotechnology, Chinese Academy of Medical Sciences \& Peking Union Medical College, Beijing 100050, China

\section{Introduction}

Nematodes are one kind of the most abundant worms and have a significant global impact on ecosystems, economies, agriculture, and human health. Plant parasitic nematodes cause huge economic losses to agriculture and forestry every year. It is of great importance to develop biological methods to control plant-parasitic nematodes, so as to deal with the environmental problems posed by chemical control methods (Duncan 1991). It has been reported that the intestinal flora in most nematodes plays an important role in host growth, physiological metabolism, and immune regulation (Nour et al. 2003; Haegeman et al. 2009). The stability of biocontrol agent activity in the field is an important factor restricting their development. Since microbiota is thought to be key to the stability of biocontrol agents, understanding the functions of the intestinal flora in nematodes is of great significance for improving the activity of biocontrol agents. 
The worm Caenorhabditis elegans is susceptible to many of the pathogens that infect plant-parasitic nematodes (Sinha et al. 2012). Like most pathogens that infect C. elegans, pathogenic bacteria colonize the digestive tract and ultimately kill the nematode. In contrast, most bacteria such as Escherichia coli and Bacillus subtilis are usually not toxic to C. elegans (Garsin et al. 2001). Thus, C. elegans has proven to be a useful and relatively simple model for studying the interactions between microbiota and pathogens. C. elegans worms are reared on bacterial cells of $E$. coli under standard laboratory conditions. Starting from early adulthood, bacterial cells colonize the intestinal lumen and re-form the entire gut microbiota (Portal-Celhay et al. 2012). However, in nature, the nematode C. elegans is a 'microbivore' because of its ability to consume various types of bacteria. To some extent, the gut microbiota of nematodes may be dominated by the bacteria that they feed on; these bacteria may shape the microbiota community structure, regulate metabolism and even alter the lifespan of the host (Han et al. 2018). In $C$. elegans, beneficial bacteria were also reported to modulate host defense responses to bacterial pathogens (Kim and Mylonakis 2012; Montalvo-Katz et al. 2013; Iatsenko et al. 2014; Dirksen et al. 2016; Berg et al. 2019; Kissoyan et al. 2019; Zimmermann et al. 2019).

In our previous study, we found that the bacterial pathogen strain "B. nematocida" B16 killed C. elegans nematodes by employing a "Trojan horse" mechanism (Niu et al. 2010). We have isolated several bacteria inside worms from various origins including soil and rotten fruit. Some bacteria, like Phytobacter sp. SCO41, showed inhibitory effects on pathogenic bacterium B16 (Wang et al. 2019). To explore the relationships between microbiota and pathogens of nematodes in-depth, we combined metagenomic sequencing analysis and culture-dependent methods to collect evidence. As a result of this analysis, we found that strain CPCC 101271, originally isolated from the intestinal lumen of C. elegans in nature, acts as a component of beneficial microbiota for $C$. elegans by extending the lifespan of the host, as well inhibiting the colonization of the host by "B. nematocida" B16, an opportunistic pathogen, which was previously proposed as a candidate biological control agent for nematodes (Huang et al. 2005). The description of the classification and identification of this strain on the basis of the polyphasic taxonomic data will be elaborated in the following article.

Here, we report an intestinal bacterium Stenotrophomonas strain CPCC 101271, and the results of in vitro and in vivo experiments showed that "B. nematocida" B16 can inhibit the growth of CPCC 101271, while strain CPCC 101271 has the ability to inhibit the colonization of C. elegans by $\mathrm{B} 16$. We also describe the variation in the microbiota community structure of $C$. elegans during competition between strain CPCC 101271 and B16.

\section{Materials and methods}

\section{Acquisition of worms and bacterial strains}

The location for screening nematodes is Baotianman Natural Reserve (33 27' 47" N; $111^{\circ} 48^{\prime} 32^{\prime \prime}$ E), Nanyang, China. Four soil samples were collected and approximately 1000 wild-living nematodes were isolated using the Baerman funnel technique (Gray 1984). Single worms were isolated and collected under a dissecting microscope. After washing three times with aseptic M9 buffer, single nematodes were frozen, ground and their crude DNA was extracted. The nematode species was identified by diagnostic PCR using the primer pair nlp30 diagnostic for C. elegans (Petersen et al. 2014). The cultivation, synchronization, collection, and surface sterilization of $C$. elegans worms were performed as previously described (Niu et al. 2012, 2015, 2016).

Strain CPCC 101271 was isolated from the surfacesterilized C. elegans worms, using Luria-Bertani (LB) agar plates. The nematodes were surface-sterilized by soaking in a solution of $1 \%$ mercuric chloride and $2 \%$ antibiotic mixture (streptomycin sulfate and gentamicin) for $1 \mathrm{~h}$, and then cultured on nutrient and oligotrophic agar plates to confirm successful surface sterilization $(0 \mathrm{cfu})$. The surface-sterilized worms were ground, then approximately $0.1 \mathrm{~g}$ of homogenate was suspended in $10 \mathrm{~mL}$ sterilized saline solution (containing $0.85 \% \mathrm{NaCl}, \mathrm{w} / \mathrm{v}$ ) and mixed thoroughly. Next, about $0.2 \mathrm{~mL}$ of suspension was spread onto an LB agar plate. After incubation at $30{ }^{\circ} \mathrm{C}$ for 2 weeks, about 40 bacterial colonies were grown on the plate. According to the colony color and size, the colonies were randomly selected for separation and purification. Among which, a distinct pale yellowish colony was picked and transferred onto a newly prepared LB agar plate for further purification. The purified isolate of CPCC 101271 was maintained as a glycerol suspension $(20 \%, \mathrm{v} / \mathrm{v})$ at $-80^{\circ} \mathrm{C}$ for long-term storage.

The reference strains Stenotrophomonas rhizophila JCM $13333^{\mathrm{T}}$ and $S$. bentonitica DSM $103927^{\mathrm{T}}$ were obtained from the Japan Collection of Microorganisms (https://jcm. brc.riken.jp/en/) and German Collection of Microorganisms and Cell Cultures (https://www.dsmz.de/collection/ catalogue), respectively. E. coli strain OP50 was obtained from the Laboratory for Conservation and Utilization of Bio-resources, Yunnan University. The opportunistic pathogen strain "B. nematocida" B16 (= GCMCC 1128) (Huang et al. 2005) was obtained from the China General Microbiological Culture Collection Center (http://www.cgmcc.net). GFP-expressing strain B16g was constructed in our previous study (Niu et al. 2012). 


\section{C. elegans lifespan assay}

Worms $C$. elegans were maintained on NGM (Nematode Growth Medium) plates at $25^{\circ} \mathrm{C}$. The strains CPCC 101271, JCM $13333^{\mathrm{T}}$ and OP50 used for measuring the worms' lifespan were recovered from the $20 \%$ glycerol stock and were streaked onto LB agar plates and then incubated at $32{ }^{\circ} \mathrm{C}$. A single colony was picked and incubated in $5 \mathrm{ml}$ of $\mathrm{LB}$ at $32{ }^{\circ} \mathrm{C}$ overnight. One milliliter of the overnight culture was added to $100 \mathrm{ml}$ of LB medium and shaken at $32{ }^{\circ} \mathrm{C}$ until an $\mathrm{OD}_{600}$ of 0.8 was reached. $200 \mu$ of the tested bacterial culture was seeded on NGM plate, and then synchronized L4 larvae were transferred to the corresponding bacterial seeded NGM plate (Park et al. 2017). The lifespan experiment was monitored by scoring the dead worms every $10 \mathrm{~h}$ from $50 \mathrm{~h}$ until $160 \mathrm{~h}$. Worms that did not respond to prodding with a platinum wire were considered dead. Those desiccated by crawling onto the edge of the housing plate were excluded from the analysis. The experiments were performed with five replicates at three different time intervals.

\section{In vitro bacteriostatic activity test}

Each bacterial strain was separately inoculated into $5 \mathrm{~mL}$ of LB medium and cultured in a shaker at $32{ }^{\circ} \mathrm{C}, 180 \mathrm{r} / \mathrm{min}$ for $12 \mathrm{~h}$. Then the culture broth was adjusted to an optical density value at $600 \mathrm{~nm}\left(\mathrm{OD}_{600}\right)$ of 1 . Approximately $0.3 \mathrm{~mL}$ of CPCC 101271 culture broth was evenly spread onto an LB agar plate. Sterilized filter paper with a diameter of $5 \mathrm{~mm}$ was immersed in the bacterial culture for $5 \mathrm{~min}$ and placed onto the agar plate containing strain CPCC 101271. The plate was then incubated at $32{ }^{\circ} \mathrm{C}$ for $48 \mathrm{~h}$, and the size of the inhibition zone for each sample was recorded. E. coli culture broth and LB medium were used as negative controls, while polymyxin B (300 IU) and rifampin $(5 \mu \mathrm{g})$ were used as positive controls. The experiments were performed with three parallels and repeated thrice.

\section{Colonization capability assay}

Colonization capability was assayed using approximately fifty 1-day-old adult hermaphrodite worms were placed on each plate at $25{ }^{\circ} \mathrm{C}$ following the procedures described by Aballay et al. (2000) and Niu et al. (2012) with modifications. In 'Feeding Transfer' experiments, the worms were transferred by hair and repeatedly washed using sterilized $\mathrm{NaCl}$ solution $(0.85 \%, \mathrm{w} / \mathrm{v})$. Three nematode treatment groups were set up. In the first group of nematodes prefed with CPCC 101271 then infected by B16g, the worms were transferred onto LB plates containing a low concentration $\left(10^{6}\right.$ cells $\left./ \mathrm{mL}\right)$ of CPCC 101271 and co-cultivated for $4 \mathrm{~h}$. The worms were then removed from the plates, washed twice, transferred to plates containing B16g and co-cultivated for $72 \mathrm{~h}$. In the second group of nematodes pre-fed with JCM $13333^{\mathrm{T}}$ then infected by B $16 \mathrm{~g}$, the worms were first seeded on an LB agar plate containing JCM13333 ${ }^{\mathrm{T}}$ $\left(10^{6}\right.$ cells $\left./ \mathrm{mL}\right)$ and cultivated for $4 \mathrm{~h}$, and then the worms were transferred to plates containing B16g and cultivated for $72 \mathrm{~h}$. In the third group, the worms were first fed on the same concentration of OP50 for $4 \mathrm{~h}$, then transferred to B 16g plates and cultivated for $72 \mathrm{~h}$. The control group of nematodes without being pre-fed with bacteria were directly seeded on a blank medium and then cultivated for $4 \mathrm{~h}$ before being transferred to $\mathrm{B} 16 \mathrm{~g}$ plates, which were also defined as B16 direct infection group. The colonization process was observed under a Nikon 800 Eclipse microscope (Nikon Corp., Japan) equipped for epifluorescence with a mercury lamp and an excitation filter of 450-490 nm (blue light) and a barrier filter of $515 \mathrm{~nm}$. At each time point, three sets of 10 nematodes were randomly selected to evaluate colonization. The worms with fluorescent bacteria in the entire lumen were scored as full; worms without any green fluorescence signal in the lumen were scored as undetected, and worms between these two extremes were scored as partial. The worms were considered dead when no movement was observed under a light-dissecting microscope, and when gently tapping of nematodes by a platinum wire, no movement occurred. Dead nematodes whose bodies were decomposed were excluded from the analysis. The number of worms killed in each group was counted every $12 \mathrm{~h}$ within $72 \mathrm{~h}$ from B16 infection. Mortality rates of B16-infected nematodes were defined as the ratio of dead nematodes to tested nematodes. The experiments were performed thrice.

\section{DNA preparation and metagenomic analysis of microbiota}

The tested nematodes were divided into five groups as follows: (I) CW00h group, which was pre-fed with CPCC 101271 for $4 \mathrm{~h}$; (II-V) CW04h, CW08h, CW12h and CW16h groups, which were separately co-cultivated with B16 for 4 , $8,12 \mathrm{~h}$ and $16 \mathrm{~h}$, respectively, after being pre-fed with CPCC 101271 for $4 \mathrm{~h}$.

The worms were collected and then washed and surface sterilized as described above. Total DNA was extracted from the intestinal microbes using the PowerSoil DNA Isolation Kit (MoBio, USA) according to the manufacturer's protocols. The concentration and purity of extracted DNA were determined using a TBS-380 and NanoDrop2000, respectively. The quality of the extracted DNA was evaluated on a $1 \%$ agarose gel. DNA was fragmented to an average size of about 300 bp using a Covaris M220 (Gene Company Limited, China) for paired-end library construction. The paired-end library was constructed using NEXTFLEX ${ }^{\circledR}$ Rapid DNA-Seq (Bioo Scientific, Austin, TX, USA). Adapters containing the full complement of sequencing primer 
hybridization sites were ligated to the blunt ends of the fragments. Paired-end sequencing was performed on an Illumina NovaSeq (Illumina Inc., San Diego, CA, USA) at Majorbio Bio-Pharm Technology Co., Ltd. (Shanghai, China) using NovaSeq Reagent Kits according to the manufacturer's instructions (www.illumina.com). Adapter sequences were stripped from the 3' and 5' ends of paired-end Illumina reads using SeqPrep (https://github.com/jstjohn/SeqPrep). Lowquality reads (length $<50 \mathrm{bp}$, a quality value $<20$, or containing $\mathrm{N}$ bases) were removed using Sickle (https://github. com/najoshi/sickle).

Metagenomics data were assembled using MEGAHIT (https://github.com/voutcn/megahit) (Li et al. 2015), which makes use of succinct de Bruijn graphs. Contigs with a length $\geq 300$ bp were selected as final assemblies and were used for further gene prediction and annotation (Noguchi et al. 2006; Li et al. 2008).

\section{Results}

\section{Increase in Caenorhabditis elegans survival rate and lifespan by feeding on CPCC 101271}

Strain CPCC 101271 was recovered from the intestinal lumen of C. elegans using LB agar plates. We compared the longevity of worms fed either on CPCC 101271, JCM $13333^{\mathrm{T}}$ or OP50. The results showed that worms fed on JCM $13333^{\mathrm{T}}$ or OP50 had almost similar lifespans. However, worms fed on CPCC 101271 lived approximately 40\% longer than worms fed on E. coli or JCM $13333^{\mathrm{T}}$, indicating that the nematodes fed on CPCC 101271 lived longer than those fed on E. coli OP50 or S. rhizophila JCM $13333^{\mathrm{T}}$ and had greatly increased survival rates (Fig. 1).

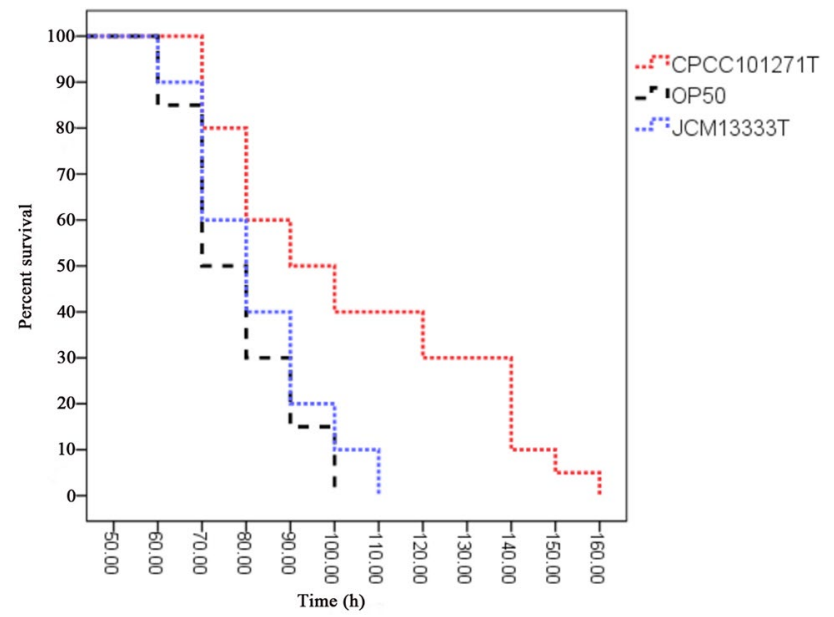

Fig. 1 Survival rates of Caenorhabditis elegans fed on different bacteria

\section{Strain CPCC 101271 confers the host with resistance to "B. nematocida" colonization}

To investigate whether strain CPCC 101271 has colonization-resistance activity against "B. nematocida" B16, we first performed an in vitro bacteriostatic activity test. The results showed that strain CPCC 101271 could not inhibit "B. nematocida" B16 but could be inhibited by B16 (Fig. 2). A transparent inhibition zone with clear edges formed around the paper containing B16 after $48 \mathrm{~h}$ of incubation on an LB agar plate spread with strain CPCC 101271. The clear zone, which was about $1.6 \mathrm{~cm}$ in diameter, was slightly smaller than the zones surrounding the positive drug controls polymyxin B (300 IU) and rifampin (5 $\mu \mathrm{g})$. And no clear inhibition zone formed around the papers containing E. coli or LB medium (Fig. 2).

GFP-expressing strain B16g was used to confirm the specificity of B16 colonization activity assays. The results of colonization-resistance activities indicated that strain CPCC 101271 could also inhibit the colonization of B16 in the nematode intestine. During the first $24 \mathrm{~h}$ of infection with "B. nematocida" B16, almost no nematodes pre-fed with CPCC 101271 were scored as being in the "full" colonization category (see Materials and Methods). By contrast, almost $20 \%$ of animals directly fed with "B. nematocida" B16 were scored as "full". In addition, after infection for $48 \mathrm{~h}, 50 \%$ of the animals fed only "B. nematocida" B16 were scored as "full". However, only $10 \%$ of worms prefed with CPCC 101271 were scored as "full" at the same time point. After $72 \mathrm{~h}$, "B. nematocida" B16 showed notably strong colonization ability, with $90 \%$ of the worms not prefed with CPCC 101271 scored as "full". In contrast, only

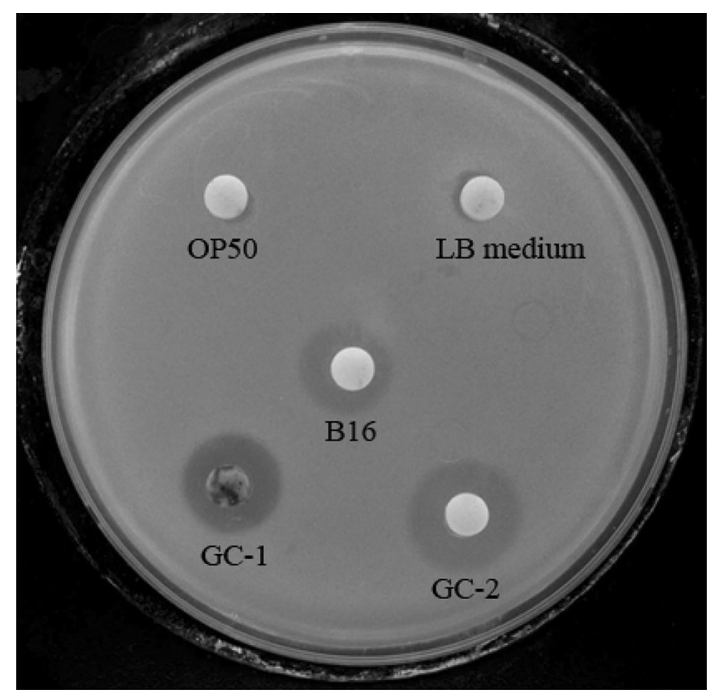

Fig. 2 Inhibitory activity of B16 against CPCC 101271 on an LB agar plate. OP50, E. coli OP50; B16, "B. nematocida" B16; GC-1, polymyxin B (300 IU); GC-2, rifampin $(5 \mu \mathrm{g})$ 
$10 \%$ of the animals pre-fed with CPCC 101271 were scored as "full". Moreover, compared with worms fed only B16, the percentage of worms fed with both B16 and CPCC 101271 that had undetectable B 16 colonization was much higher at $48 \mathrm{~h}(10 \%$ vs. $80 \%)$ and at $72 \mathrm{~h}(0 \%$ vs. $70 \%)$. The worms pre-fed with $E$. coli showed little difference compared with those in the no pre-feeding group. The ability of B16 to colonize the nematodes pre-fed with JCM $13333^{\mathrm{T}}$ was stronger than its ability to colonize those pre-fed with CPCC 101271, but a little weaker than its ability to colonize the negative controls pre-fed with $E$. coli OP50. Differences between the abilities of B16 to colonize the nematodes pre-fed with CPCC 101271 and pre-fed with OP50 were notable when we compared the changes in the severity of colonization at 72-h (Fig. 3, chi-squared test, $P<0.0001$ ). For example, only $10 \%$ of worms that were pre-fed with CPCC 101271 could be categorized as having 'full' colonization. However, $90 \%$ of worms that were pre-fed with OP50 were categorized in the 'full' colonization category. The results indicated that colonization of "B. nematocida" B16 was markedly attenuated in C. elegans pre-fed with CPCC 101271.

The differences in the mortalities of the B16-infected nematodes in the different treatment groups indicated that pre-feeding with CPCC 101271 reduced the mortality caused by infection with the pathogenic bacteria B16 (Fig. 4). The mortalities of the nematodes pre-fed with OP50 and then infected by B16 and the nematodes directly infected by B16 (without pre-feeding with any other bacteria) were 85 and $90 \%$ within $60 \mathrm{~h}$, respectively. By contrast, for worms prefed with CPCC 101271 then infected with B16, the mortality dropped to $40 \%$. The natural mortality rate of the negative control nematodes (no pre-feeding or B16 infection) was only $18 \%$. At other time points, the mortalities of nematodes pre-fed with CPCC 101271 were significantly lower than those of nematodes pre-fed with $E$. coli or directly infected with B16.

\section{The variation in Caenorhabditis elegans microbiota community structure during competition between CPCC 101271 and B16}

In a previous study, we collected free-living terrestrial $C$. elegans from soil and rotten fruits, and analyzed the variation in intestinal flora following "B. nematocida" B16 infection by performing macrogenomic analysis. We found significant differences in the diversity and distribution of microbiota between the control worms and those infected with B16 for $24 \mathrm{~h}$. The diversity of the intestinal microbiome decreased after B16 infection (Niu et al. 2016). Here we aimed to investigate the variation in the intestinal bacterial community structure of $C$. elegans during competition between CPCC 101271 and B16.

A total of $332,314,280,966,705,161,227,126$ and 597,664 sequences comprising 44, 26, 49, 19 and 30 operational taxonomic units (OTUs) were obtained from the five groups CW00h, CW04h, CW08h, CW12h, and CW16h, respectively. At the genus level, these OTUs represented 26, 14, 23, 10, and 14 genera, respectively. It was obvious that the bacteria diversity was greatly decreased during competition between CPCC 101271 and B16 (Fig. 5). At the first stage of infection (CW00h) in nematodes pre-fed with strain CPCC 101271, the microbiota community structure predominantly consisted of the genera Bacillus, Acetobacter, Lactobacillus, Phytobacter, Stenotrophomonas, Pichia
Fig. 3 Differences in " $B$. nematocida" B16 colonization of C. elegans after 24, 48, and $72 \mathrm{~h}$ of infection. For each bacterial strain tested, the extent of colonization was scored in four sets of 10 nematodes every $24 \mathrm{~h}$. A representative of three independent experiments with the average fraction of the population colonized for each category is shown. Chi-squared test, $* P<0.05, * * P<0.001$. CPCC 101271, Stenotrophomonas strain CPCC 101271; JCM $13333^{\mathrm{T}}$, Stenotrophomonas rhizophila $\mathrm{JCM} 13333^{\mathrm{T}}$; OP50, E. coli OP50

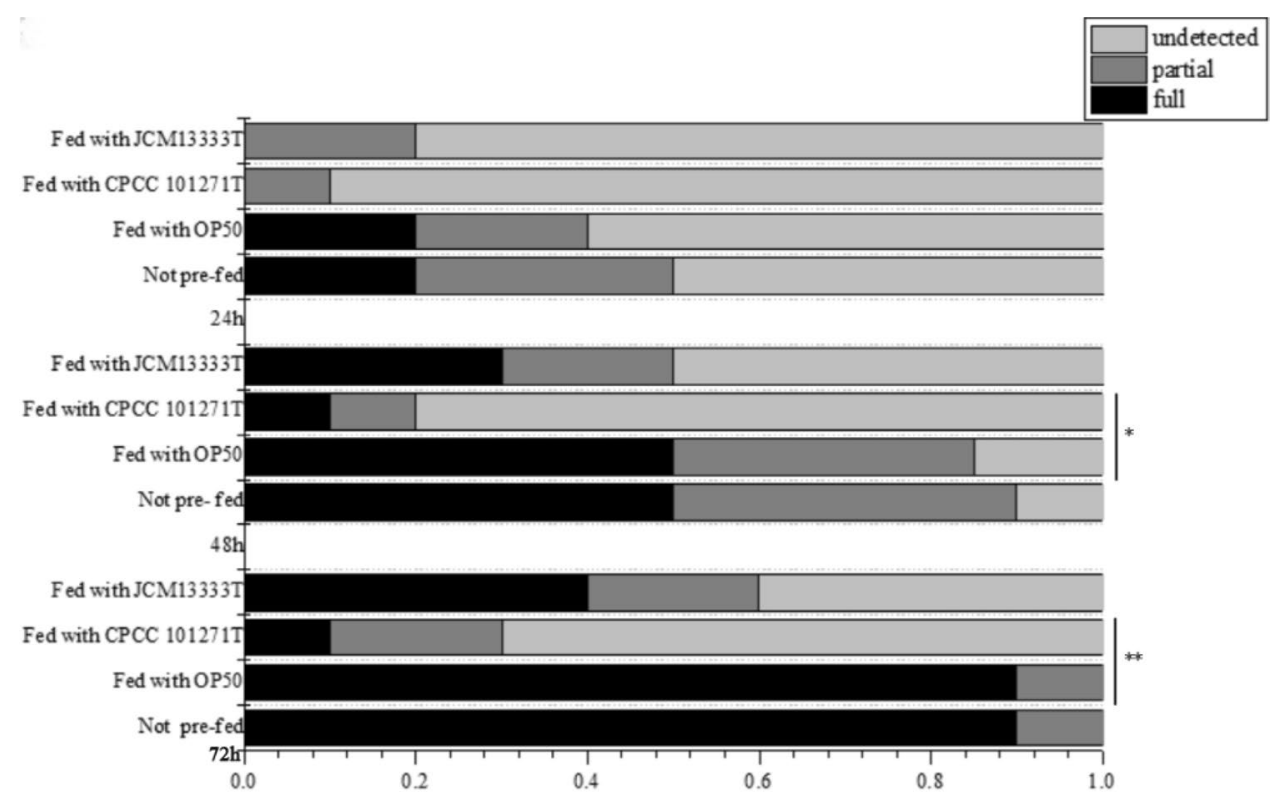

Proportion of nematodes with different levels of B16 colonization 
Fig. 4 The differences in mortality rates of B16-infected nematodes in different treatment groups. CPCC 101271, Stenotrophomonas strain CPCC 101271; JCM $13333^{\mathrm{T}}$, Stenotrophomonas rhizophila JCM $13333^{\mathrm{T}}$; B16, "Bacillus nematocida" B16; OP50, Escherichia coli $\mathrm{OP} 50$
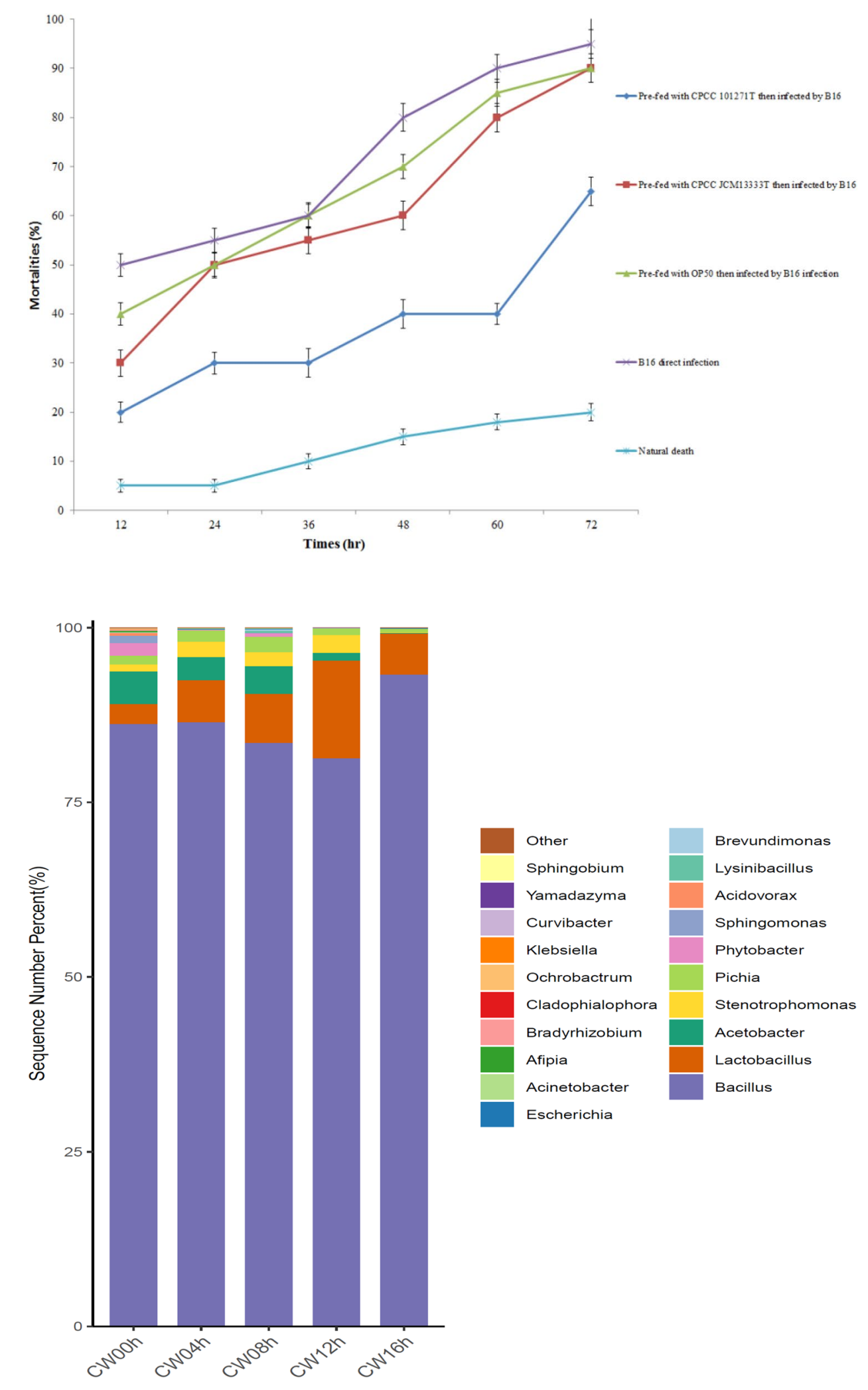

Fig. 5 Column diagram showing the microbiota structure at the genus level, based on metagenomic sequence analysis, in nematodes pre-fed with CPCC 101271 before and after being infected by B16. CW00h, pre-fed with CPCC 101271 for 4 h; CW04h, CW08h, CW12h and CW16h, groups co-cultured with B16 for $4,8,12$, and $16 \mathrm{~h}$, respectively, after being pre-fed with CPCC 101271 for $4 \mathrm{~h}$ and Sphingomonas. At the second stage (CW04h), $4 \mathrm{~h}$ after the worms were infected by B16, dysbiosis occurred, and in the course of re-construction of the microbiota community, the bacteria diversity was drastically reduced. Besides
Lactobacillus spp., Acetobacter spp. and Pichia spp., which remained the major groups, the abundance of Bacillus spp. increased slightly and the abundance of Stenotrophomonas spp. increased. At the third stage (CW08h), which we termed 
"the breaking period", a large number of CPCC 101271 and B16 bacteria were co-existing and competing; the diversity of the intestinal flora had partially recovered, but the abundance of Bacillus spp. had greatly decreased. At the fourth stage (CW12h), the abundance of Bacillus spp. was even lower and the abundance of Stenotrophomonas spp. was higher. The newly reconstructed intestinal flora was disrupted again, and the species composition was the most similar to that observed at the second stage (Fig. 6). By the fifth stage (CW16h), B16 overwhelmed CPCC 101271 and only Lactobacillus spp. and Pichia spp., together with Bacillus spp., remained the major microbiota.
In a summary, over the course of B16 infection, the abundance of CPCC 101271 and its relatives (Stenotrophomonas spp.) kept on increasing until $8 \mathrm{~h}$ after B16 infection and then decreased sharply. The change in abundance of Stenotrophomonas spp. was accompanied by similar changes in the abundance of alpha-trehalose-phosphate synthase-encoding genes calculated from the metagenome data, except at the final stage (Figure S1). By contrast, the abundance of Bacillus spp. first decreased, then increased rapidly, which was similar to the changes in the abundance of trehalose6-phosphate hydrolase-encoding genes (Figure S2). The abundances of alpha-trehalose-phosphate synthase-encoding
Fig. 6 Heatmap based on metagenomic sequence analysis showing the microbiota structure at the genus level in nematodes pre-fed with CPCC 101271 before and after being infected by B16

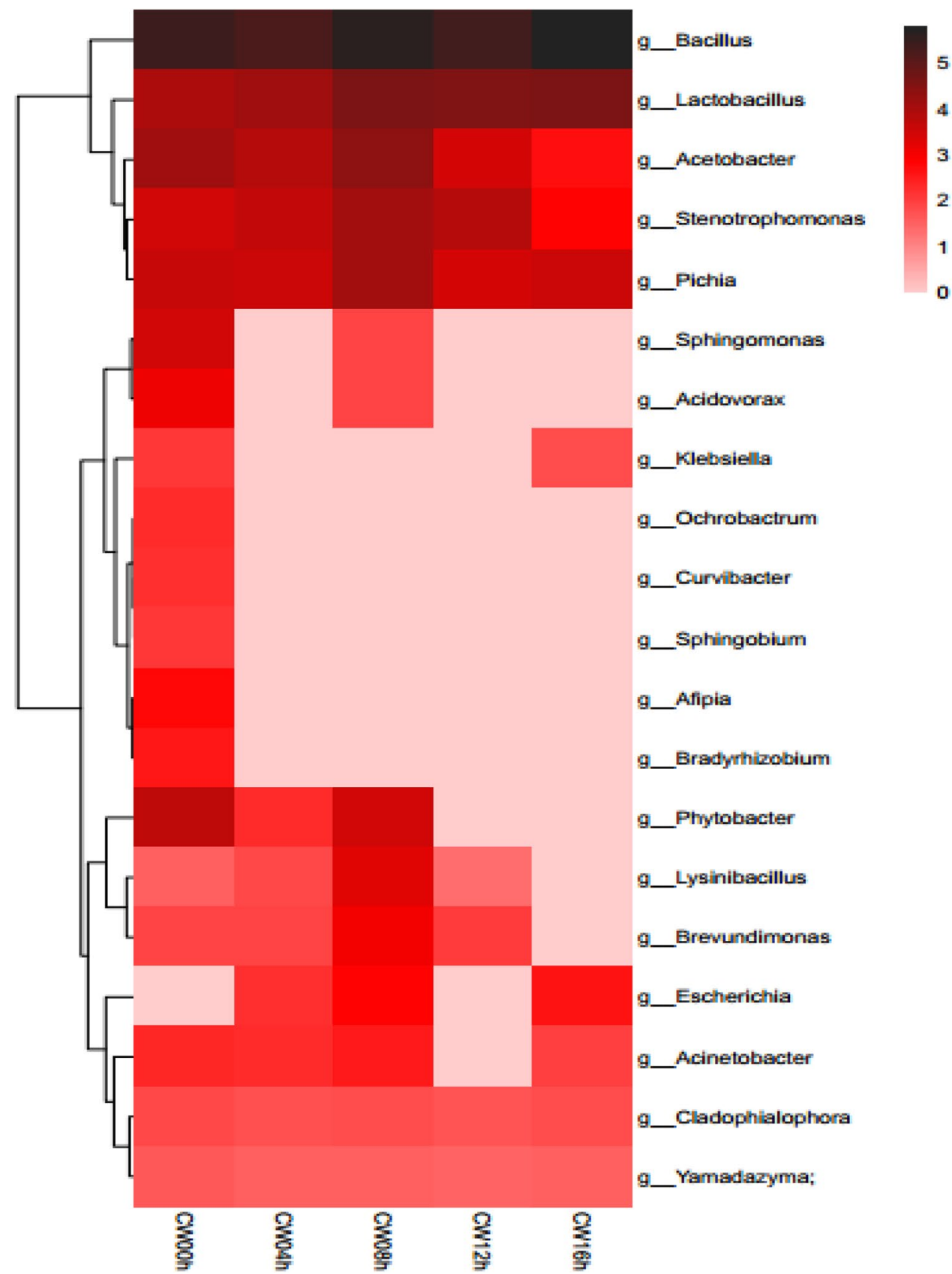


genes (present in the genome of B16) and trehalose-6-phosphate hydrolase-encoding genes (present in the genome of CPCC 101271 ) together with Stenotrophomonas spp. and Bacillus spp. reached the highest level at $8 \mathrm{~h}$ (CW08h) after B16 infection. The results suggested that strain CPCC 101271 possibly participated directly or induced some other bacteria in the community to participate in resistance to B16 colonization. However, by $16 \mathrm{~h}$ after infection, B16 dominated the microbiota community, and the growth of CPCC 101271 was completely suppressed.

\section{Discussion}

Gut microbiota, diverse microorganisms inhabiting the digestive track, are tightly linked to the health of their host. The community of microbial species, among which bacteria are predominant and have been extensively studied, not only generates metabolites essential for various host functions but also confers resistance to exogenous pathogens (Lee and Hase 2014). However, the molecular mechanisms by which microbiota resist pathogens and the changes in bacterial composition that occur after pathogen infection remains elusive.

It has been reported that bacteria living in most nematodes play an important role in the growth and development, physiological metabolism, and immune regulation of the host. The bacteria Xenorhabdus spp. and Photorhabdus spp. were reported to be symbionts found in the guts of nematodes including Steinernema, Heteronrhahditis, Heterodera and Rhabditis. They produce antibiotics, intracellular protein crystals, and numerous other products that help nematodes kill insects and also provide nutrients (Forst et al. 1997; Park et al. 2011; Whittaker et al. 2016; Shan et al. 2019). Bacteria associated with cysts of the soybean cyst nematode play an important ecological role in the long-term survival of cysts in soil (Nour et al. 2003). An endo-symbiotic bacterium in a plant-parasitic nematode was found to be closely associated with the growth and metabolism of its host (Haegeman et al. 2009). Therefore, to some extent, nematodes are typical symbioses with their microbiota.

For nematodes C. elegans, most of the research work was carried out based on the N2 strain, which has been adapted to laboratory conditions over decades (Sterken et al. 2015), including the regular and routine removal of any microbes through hypochlorite treatment. Thus N2 strain does not carry any microbes in its gut and microbiome associations are little known to the nematode $C$. elegans N2 under laboratory conditions. In contrast, worms in nature are exposed to complex microbial communities. Understanding the worm's natural microbiome is essential to help explain their realistic and unbiased characteristics. In fact, more and more researchers have paid their attention to the natural $C$. elegans microbiome (Dirksen et al. 2016; Samuel et al. 2016; Zhang et al. 2017). A possible fitness benefit was already indicated upon gut colonization with certain non-pathogenic bacteria, leading to increased resistance against pathogens (Ikeda et al. 2007; Kim and Mylonakis 2012; Montalvo-Katz et al. 2013). However, it is yet unclear whether the beneficial bacterial isolates in C. elegans affect the infection effect of pathogenic bacteria on nematodes. The activities and stability of biological control agents might be effectively improved by using co-cultures of various antagonistic bacteria with different mechanisms of action and ecological adaptability. Therefore, using natural C. elegans as a model, studying the interactions between microbiota and biocontrol microbes is a promising approach for improving the stability of biocontrol in the field.

To date, there are 16 validly described species in the genus Stenotrophomonas, which have high genotypic and phenotypic diversity and were recovered from various environmental and even clinical samples (Brooke 2012). The type species $S$. maltophilia was originally recognized as a human opportunistic pathogen. Subsequent research revealed that the metabolic diversity of $S$. maltophilia is responsible for the production of novel bioactive compounds, including biocontrol agents against microbes and insects, and enzymes and nanoparticles used in medicinal, industrial, and bioremediation applications (Ribitsch et al. 2012). Another well-studied species, S. rhizophila, which shows an endophytic lifestyle, possesses unique genes encoding plant cell-wall-degrading enzymes and proteins responsible for the synthesis and transport of the plant-protective spermidine and high salinity tolerance, which suggests it is a harmless alternative Stenotrophomonas species for use in biotechnology (Alavi et al. 2013). There were also several reports on the genus Stenotrophomonas strains isolated from animal intestines. Stenotrophomonas members were found to be gut bacteria through the life cycle of the Bark Beetle Dendroctonus rhizophagus, and S. maltophilia could be implicated in nitrogen fixation and cellulose breakdown, important roles associated to insect development and fitness, especially under the particularly harsh life conditions of this beetle (Morales-Jiménez et al. 2012). Additionally, Sun et al. isolated a chitin-degradation Stenotrophomonas strain from the hindgut of a fungus-growing termite Macrotermes barneyi (Sun et al. 2017). The novel species studied here, a close relative of $S$. rhizophila, was generally consistent with those previously reported Stenotrophomonas species isolated from the nematodes sampled directly from the native habitats (Dirksen et al. 2016). In each parallel of the isolation experiment, we selected randomly 20 single natural worms isolated from the same location. Members of the genus Stenotrophomonas could be isolated from more than 15 worms. The Stenotrophomonas spp. were identified to be the same species with CPCC 101271. Furthermore, 
other worms without Stenotrophomonas being detected were raised on 9-cm agar plates seeded with $400 \mu \mathrm{l}$ of the tested bacterium CPCC 101271 with an $\mathrm{OD}_{600}$ of 10 for $24 \mathrm{~h}$ at room temperature. Then Stenotrophomonas spp. could be isolated from the intestines of the worms after washed three times and surface disinfection. These experiments indicated that CPCC 101271 could stably colonize the nematode gut under experimental conditions.

In nature, the structure and diversity of the microbiota in healthy nematodes are constantly changing. Some intestinal bacteria are actually indispensable parts of the host, which may form a mutually beneficial symbiotic relationship with the host.

In this study, the interaction between the bacterium CPCC 101271 and "B. nematocida" B16 was investigated. When strains B16 and CPCC 101271 were co-cultured on LB plates, strain B16 showed inhibitory activities against CPCC 101271, which is consistent with the last stage of the competition between strain CPCC 101271 and B16 in worm intestine, even strain CPCC 101271 exhibited the colonization-resistance activities against "B. nematocida" B16 in the early stages. Firstly, strain CPCC 101271 was confirmed as probiotic to worms owning to its ability to prolong the lifespan of C. elegans. Secondly, strain CPCC 101271 could delay the infection time of B16 against nematodes, but not completely inhibit the infection of B16. Last but not least, Bacillus strains occupied the niche of Stenotrophomonas members by inhibiting the growth of CPCC 101271, which could be inferred from the metagenomic analysis results. And then strain B16 completed the infection and realized its proliferation in worms.

Based on the above experiments, we proposed that the reason for colonization resistance to the pathogen $\mathrm{B} 16$ by the strain CPCC 101271 might own to its beneficial aspects to $C$. elegans. Strain CPCC 101271 might play a critical role in (i) shaping and maintaining the intestinal bacterial community structure, (ii) synthesizing osmoprotectants, such as glucosyl glycerol and trehalose, to help maintain host homeostasis, and (iii) producing or stimulating other microorganisms to synthesize antimicrobial peptides and other stress-protective agents to protect the host from pathogens and harsh environments.

We should explore substantial evidence to confirm the above inference in the following studies. Understanding this inference mechanism can help quickly inhibit the growth of probiotic microbiota, accelerate the colonization of biocontrol bacteria in the intestinal tract and improve the killing efficiency of nematodes. Our current findings may lay a theoretical foundation and open up new ideas for the development of ideal biocontrol agents.

Supplementary Information The online version contains supplementary material available at https://doi.org/10.1007/s00203-021-02467-4.
Acknowledgements The authors thank JCM (Japan Collection of Microorganisms) and DSMZ (German Collection of Microorganisms and Cell Cultures) for providing the reference strains.

Authors' contributions RH and YW carried out the experiments. YD prepared the figures. YZ and LZ designed the project and prepared the manuscript together with QN. All authors reviewed the manuscript.

Funding This research was supported by the National Natural Science Foundation of China (NSFC 31670010 and 31570120), the Drug Innovation Major Project (2018ZX09711001-007-001), the National Infrastructure of Microbial Resources (NIMR-2020-3), CAMS Innovation Fund for Medical Sciences (CIFMS) (2016-I2M-2-002) and Innovation Scientists and Technicians Troop Construction Projects (Sustainable Utilization of Energy Microbial Resources) of Henan Province.

Data availability The DDBJ/EMBL/GenBank accession number for the 16S rRNA gene sequence of strain CPCC 101271 is MT126327; The draft genome sequence of strain CPCC 101271 is WIAY00000000. The strain CPCC 101271 has been deposited in China Pharmaceutical Culture Collection and is available to the scientific research community without any special restrictions.

\section{Declarations}

Conflict of interest The authors declare that the research was conducted in the absence of any commercial or financial relationships that could be construed as a potential conflict of interest.

Open Access This article is licensed under a Creative Commons Attribution 4.0 International License, which permits use, sharing, adaptation, distribution and reproduction in any medium or format, as long as you give appropriate credit to the original author(s) and the source, provide a link to the Creative Commons licence, and indicate if changes were made. The images or other third party material in this article are included in the article's Creative Commons licence, unless indicated otherwise in a credit line to the material. If material is not included in the article's Creative Commons licence and your intended use is not permitted by statutory regulation or exceeds the permitted use, you will need to obtain permission directly from the copyright holder. To view a copy of this licence, visit http://creativecommons.org/licenses/by/4.0/.

\section{References}

Aballay A, Yorgey P, Ausubel FM (2000) Salmonella typhimurium proliferates and establishes a persistent infection in the intestine of Caenorhabditis elegans. Curr Biol 10:1539-1542

Alavi P, Starcher MR, Zachow C, Muller H, Berg G (2013) Rootmicrobe systems: the effect and mode of interaction of Stress Protecting Agent (SPA) Stenotrophomonas rhizophila DSM14405(T.). Front Plant Sci 4:141

Berg M, Monnin D, Cho J, Nelson L, Crits-Christoph A, Shapira M (2019) TGF $\beta / B M P$ immune signaling affects abundance and function of C. elegans gut commensals. Nat Commun 10:604

Brooke JS (2012) Stenotrophomonas maltophilia: an emerging global opportunistic pathogen. Clin Microbiol Rev 25:2-41

Dirksen P, Marsh SA, Braker I, Heitland N, Wagner S, Nakad R, Mader S, Petersen C, Kowallik V, Rosenstiel P (2016) The native microbiome of the nematode Caenorhabditis elegans: gateway to a new host-microbiome model. BMC Biol 14:38

Duncan LW (1991) Current options for nematode management. Annu Rev Phytopathol 29:469-490 
Forst S, Dowds B, Boemare N, Stackebrandt E (1997) Xenorhabdus and Photorhabdus spp.: bugs that kill bugs. Annu Rev Microbiol 51:47-72

Garsin DA, Sifri CD, Mylonakis E, Qin X, Singh KV, Murray BE, Calderwood SB, Ausubel FM (2001) A simple model host for identifying gram-positive virulence factors. P Natl Acad Sci USA 98:10892-10897

Gray NF (1984) Ecology of nematophagous fungi: comparison of the soil sprinkling method with the Baerman funnel technique in the isolation of endoparasites. Soil Biol Biochem 16:81-83

Haegeman A, Vanholme B, Jacob J, Vandekerckhove TT, Claeys M, Borgonie G, Gheysen G (2009) An endosymbiotic bacterium in a plant-parasitic nematode: member of a new Wolbachia supergroup. Int J Paraitol 39:1045-1054

Han B, Sivaramakrishnan P, Lin CJ, Neve IA, He J, Tay LW, Sowa JN, Sizovs A, Du G, Wang J, Herman C, Wang MC (2018) Microbial genetic composition tunes host longevity. Cell 173:1058

Huang XW, Niu QH, Zhou W, Zhang KQ (2005) "Bacillus nematocida" sp. nov., a novel bacterial strain with nematotoxic activity isolated from soil in Yunnan. China Syst Appl Microbiol 28:323-327

Iatsenko I, Yim JJ, Schroeder FC, Sommer RJ (2014) B. subtilis GS67 protects $C$. elegans from Gram-positive pathogens via fengycinmediated microbial antagonism. Curr Biol 24:2720-2727

Ikeda T, Yasui C, Hoshino K, Arikawa K, Nishikawa Y (2007) Influence of lactic acid bacteria on longevity of Caenorhabditis elegans and host defense against Salmonella enterica Serovar Enteritidis. Appl Environ Microb. https://doi.org/10.1128/AEM. 00704-07

Kim Y, Mylonakis E (2012) Caenorhabditis elegans immune conditioning with the probiotic bacterium Lactobacillus acidophilus strain NCFM enhances gram-positive immune responses. Infect Immun 80:2500-2508

Kissoyan KA, Drechsler M, Stange EL, Zimmermann J, Kaleta C, Bode HB, Dierking K (2019) Natural C. elegans microbiota protects against infection via production of a cyclic lipopeptide of the viscosin group. Curr Biol 29:1030-1037.e5

Lee WJ, Hase K (2014) Gut microbiota-generated metabolites in animal health and disease. Nat Chem Biol 10:416-424

Li R, Li Y, Kristiansen K, Wang J (2008) SOAP: short oligonucleotide alignment program. Bioinformatics 24:713-714

Li D, Liu CM, Luo R, Sadakane K, Lam TW (2015) MEGAHIT: an ultra-fast single-node solution for large and complex metagenomics assembly via succinct de Bruijn graph. Bioinformatics $31: 1674-1676$

Montalvo-Katz S, Huang H, Appel MD, Berg M, Shapira M (2013) Association with soil bacteria enhances p38-dependent infection resistance in Caenorhabditis elegans. Infect Immun 81:514-520

Morales-Jiménez J, Zúñiga G, Ramírez-Saad H, Hernández-Rodríguez C (2012) Gut-associated bacteria throughout the life cycle of the bark beetle Dendroctonus rhizophagus thomas and bright (Curculionidae: Scolytinae) and their cellulolytic activities. Microb Ecol 64:268-278

Niu Q, Huang X, Zhang L, Xu J, Yang D, Wei K, Niu X, An Z, Bennett J, Zou C, Yang J, Zhang K (2010) A Trojan horse mechanism of bacterial pathogenesis against nematodes. Proc Natl Acad Sci USA 107:16631-16636

Niu Q, Huang X, Hui F, Huang S, Ke T, Zhang K, Zhang L (2012) Colonization of Caenorhabditis elegans by "Bacillus nematocida" B16, a bacterial opportunistic pathogen. J Mol Microb Biotech 22:258-267

Niu Q, Zheng H, Zhang L, Qin F, Facemire L, Zhang G, Cao F, Zhang K, Huang X, Yang J, He L, Liu C (2015) Knockout of the $a d p$ gene related with colonization in "Bacillus nematocida" B16 using customized transcription activator-like effectors nucleases. Microb Biotechnol 8:681-692
Niu Q, Zhang L, Zhang K, Huang X, Hui F, Kan Y, Yao L (2016) Changes in intestinal microflora of Caenorhabditis elegans following "Bacillus nematocida" B16 infection. Sci Rep 6:20178

Noguchi H, Park J, Takagi T (2006) MetaGene: prokaryotic gene finding from environmental genome shotgun sequences. Nucleic Acids Res 34:5623-5630

Nour SM, Lawrence JR, Zhu H, Swerhone GD, Welsh M, Welacky TW, Topp E (2003) Bacteria associated with cysts of the soybean cyst nematode (Heterodera glycines). Appl Environ Microb 69:607-615

Park HE, Jung Y, Lee SJ (2017) Survival assays using Caenorhabditis elegans. Mol Cells 40:90-99

Park HW, Kim YO, Ha JS, Youn SH, Kim HH, Bilgrami AL, Shin CS (2011) Effects of associated bacteria on the pathogenicity and reproduction of the insect-parasitic nematode Rhabditis blumi (Nematoda: Rhabditida). Can J Microbiol 57:750-758

Petersen C, Dirksen P, Prahl S, Strathmann E, Schulenburg H (2014) The prevalence of Caenorhabditis elegans across 1.5 years in selected North German locations: the importance of substrate type, abiotic parameters, and Caenorhabditis competitors. BMC Ecol 14:4

Portal-Celhay C, Bradley ER, Blaser MJ (2012) Control of intestinal bacterial proliferation in regulation of lifespan in Caenorhabditis elegans. BMC Microbiol 12:49

Ribitsch D, Heumann S, Karl W, Gerlach J, Leber R, Birner-Gruenberger R, Gruber K, Eiteljoerg I, Remler P, Siegert P, Lange J, Maurer KH, Berg G, Guebitz GM, Schwab H (2012) Extracellular serine proteases from Stenotrophomonas maltophilia: Screening, isolation and heterologous expression in E. coli. J Biotechnol 157:140-147

Samuel BS, Rowedder H, Braendle C, Félix M, Ruvkun G (2016) Caenorhabditis elegans responses to bacteria from its natural habitats. Proc Natl Acad Sci USA 113:E3941-E3949

Shan SJ, Wang WW, Song CX, Bingjiao M, Yang S (2019) The symbiotic bacteria Alcaligenes faecalis of the entomopathogenic nematodes Oscheius spp. exhibit potential biocontrol of plant and entomopathogenic fungi. Microb Biotechnol 12:459-471

Sinha A, Rae R, Iatsenko I, Sommer RJ (2012) System wide analysis of the evolution of innate immunity in the nematode model species Caenorhabditis elegans and Pristionchus pacificus. PLoS One 7:e44255

Sterken MG, Snoek LB, Kammenga JE, Andersen EC (2015) The laboratory domestication of Caenorhabditis elegans. Trends Genet $31: 224-231$

Sun XX, Li JJ, Ning N, Tan HJ, Jin-Feng NI (2017) Isolation and identification of chitin-degrading bacteria from the hindgut of Macrotermes barneyi. Microbiol China 44:1649-1654

Wang B, Huang B, Chen J, Li W, Yang L, Yao L, Niu Q (2019) Wholegenome analysis of the colonization-resistant bacterium Phytobacter sp. SCO41(T) isolated from "Bacillus nematocida" B16-fed adult Caenorhabditis elegans. Mol Biol Rep 46:1563-1575

Whittaker JH, Robertson A, Kimber MJ, Day T, Carlson S (2016) Intestinal Enterobacteriaceae that protect nematodes from the effects of benzimidazoles. J Bacteriol Parasitol 7:05

Zhang F, Berg M, Dierking K, Félix M, Shapira M, Samue BS, Schulenburg H (2017) Caenorhabditis elegans as a model for microbiome research. Front Microbiol 8:485

Zimmermann J, Obeng N, Yang W, Pees B, Petersen C, Waschina S, Kissoyan K, Aidley J, Hoeppner M, Bunk B, Spröer C, Leippe M, Dierking K, Kaleta C, Schulenburg H (2019) The functional repertoire contained within the native microbiota of the model nematode Caenorhabditis elegans. ISME J 14:26-38

Publisher's Note Springer Nature remains neutral with regard to jurisdictional claims in published maps and institutional affiliations. 